\section{Diseño y validación de un instrumento de medición de la carga académica de estudiantes de carreras de la salud}

\author{
NATALIA HARDEN DÍAZ $Z^{1, a}$, CONSTANZA PINO LEÓN ${ }^{1, b}$, \\ SHENDA ORREGO MOLINA ${ }^{1, \mathrm{c}}$, SANDRA FLORES ALVARADO ${ }^{1,3, \mathrm{~d}}$, \\ JAVIERA ESTURILLO PINET ${ }^{1, \mathrm{e}}$, VALENTINA FLORES CASTRO ${ }^{1, \mathrm{e}}$, \\ GABRIELA RUBIO CALERO ${ }^{2, f}$, BÁRBARA AGUIAR ARIAS ${ }^{2, \mathrm{f}}$, \\ JOSÉ PERALTA CAMPOSANO ${ }^{1,2, g}$
}

\section{Design of a real and perceived academic workload measurement instrument for health care undergraduate students}

Background: A high academic workload may have adverse consequences among university students. Aim: To design and validate an instrument to measure both real and perceived academic workload for health care students. Material and Methods: The questionnaire was designed based on a bibliographic revision and the conduction of two focus groups conformed by undergraduate students from a Faculty of Medicine. Afterwards, it was submitted to qualitative pre-tests. The final instrument consists of a self-applied questionnaire with both a characterization section (10 questions) and one concerning academic workload by subject and semester (five and two questions, respectively). A national and international panel of 14 experts evaluated the survey content's validity. The analysis was performed according to the Content Validity Ratio and the Content Validity Index. Results: The complete instrument was validated with an $84 \%$ consensus between the judges. Each section of the instrument was approved separately with a 77\% and a 94\% agreement, respectively. After being individually analyzed by the judges, each question was validated. The wording of questions was improved taking the experts comments into consideration. Conclusions: The proposed instrument constitutes a contribution for the measurement of real and perceived academic workload for students.

(Rev Med Chile 2021; 149: 873-880)

Key words: Students, Health Occupations; Surveys and Questionnaires; Validation Study; Workload.

\author{
'Dirección Académica, Facultad \\ de Medicina, Universidad de \\ Chile, Santiago, Chile. \\ ${ }^{2}$ Dirección de Pregrado, Facultad \\ de Medicina, Universidad de \\ Chile, Santiago, Chile. \\ ${ }^{3}$ Programa de Bioestadística, \\ Instituto de Salud Poblacional, \\ Universidad de Chile, Santiago, \\ Chile. \\ asocióloga. \\ ${ }^{\mathrm{b} A n t r o p o ́ l o g a ~ F i ́ s i c a ; ~ E s t u d i a n t e ~}$ \\ de Magíster en Bioestadística. \\ 'Máster en Evaluación y \\ entrenamiento físico para la \\ salud. \\ dAntropóloga Física; Magíster \\ en Bioestadística; Estudiante de \\ Doctorado en Salud Pública. \\ eLicenciada en Sociología. \\ Terapeuta Ocupacional. \\ 9Magíster en Educación en \\ Ciencias de la Salud. \\ Trabajo no recibió \\ financiamiento. \\ Los autores declaran no tener \\ conflictos de interés.
}

Recibido el 23 de noviembre de 2020, aceptado el 13 de abril de 2021.

Correspondencia a: José Peralta Camposano Avenida Independencia 1027, Independencia, Santiago, Chile. jperalta@uchile.cl
L a carga de trabajo de las y los estudiantes de educación superior, o carga académica, se ha $\checkmark$ perfilado como un elemento relevante a la hora de gestionar los planes de formación en instituciones de educación terciaria, ya que afecta tanto su permanencia en el sistema como su desempeño académico y proceso de aprendizaje en general ${ }^{1-6}$. Diversos autores afirman que un exceso de carga académica puede tener consecuencias académicas como el rezago, además de efectos sobre la salud mental: depresión, ansiedad, burnout, problemas de autoestima, entre otros ${ }^{1,2,7-9}$. Una serie de investigaciones afirman que los estudiantes de carreras de la salud son propensos a este tipo de afecciones asociadas a la carga académica como inseguridad en la aproximación al estudio, pánico y estrés grupal y falta de confianza $a^{7,10,11}$.

A nivel internacional han surgido diferentes 
iniciativas para regular la cantidad de horas de trabajo de los estudiantes, destacándose la Declaración de Bolonia en $1999^{12}$. A nivel nacional, en el año 2006 se elaboró e implementó el Sistema de Créditos Transferibles (SCT) de acuerdo al European Credit Transfer System (ECTS) con el objetivo, entre otros, de promover la armonización curricular ${ }^{12,13}$.

Pese a esto, la utilización de créditos no ha logrado resolver el problema, debido a que existe discrepancia entre la carga académica declarada en los planes de estudio (en la forma de créditos asignados a cada asignatura) y el tiempo que los estudiantes ocupan efectivamente dentro y fuera del horario de clase: la carga académica real ${ }^{14}$.

Esto refleja que la carga académica es un problema complejo y dinámico, lo que tiene como consecuencia que no exista una única aproximación a su estudio y medición. El número de horas trabajadas en una semana típica constituyó por años el indicador más utilizado para medirla ${ }^{3,15,16}$, sin embargo, se ha evidenciado que su uso puede traer consigo problemas como la fiabilidad de los datos brindados por los estudiantes, como grandes diferencias para una misma asignatura entre quienes son consultados ${ }^{17}$. Ya en 1992, Chambers reconocía la dificultad de preguntar las horas dedicadas a estudiar, dado que muchos factores pueden llegar a influenciar esta estimación ${ }^{2}$.

Además de los eventuales problemas metodológicos asociados a la medición de las horas trabajadas, investigaciones sobre resultados de aprendizaje argumentan que las percepciones del estudiantado deben ser tomadas en cuenta con mayor preponderancia que la medida objetiva de las variables ${ }^{18-22}$. Así comienza una corriente de estudio de la carga académica que centra su foco de investigación en la medición de la carga percibida, correspondiente a la percepción que tiene el estudiante de las demandas académicas puestas sobre él ${ }^{18,24,25}$.

La carga percibida se ve influenciada por un conjunto de variables o factores interrelacionados, dentro de los cuales la carga real no constituye el único factor relevante ${ }^{1}$. De hecho, Kember determinó, en 2006, que no existe necesariamente una relación entre las horas de trabajo real de los estudiantes y la carga académica percibida por ellos. Esto deja en evidencia que el tiempo invertido no es suficiente por sí mismo para estudiar este fenómeno, haciendo necesario adentrarse en el estudio de los factores que afectan las percepciones de carga académica del estudiantado ${ }^{24-26}$.

Dado lo anteriormente expuesto, se considera relevante contar con un instrumento que mida la carga académica real y percibida y que, además, recoja los factores que podrían influir en la percepción de carga académica del estudiantado, dando nuevas luces sobre la relación entre la carga real y la declarada en los programas de estudio; y entre la carga real y la carga percibida.

La presente investigación se contextualiza en el proceso de revisión y actualización de los procesos de innovación curricular realizado en la Facultad de Medicina de la Universidad de Chile (FMUCH). En el contexto local, se hace relevante estudiar la carga real y percibida, tanto para apoyar la evaluación de la implementación de los SCT concluida en 2013 en las 8 carreras de la facultad, como para evitar los efectos adversos de la sobrecarga que han sido evidenciados en estudiantes de carreras de la salud. Así, el objetivo del presente trabajo fue diseñar y validar un instrumento que permitiera medir carga académica real y percibida en estudiantes de educación superior del área de salud.

\section{Materiales y Métodos}

Para la construcción del cuestionario se realizó una revisión bibliográfica que incluyó los conceptos "workload", "overload", "academic workload", "academic overload", "perceived workload" y "student workload" en las bases de datos Google académico, ERIC, Education Source, Scopus, EBSCOhost, ISI e International Journal of Inclusive Education, sin delimitación del año de publicación de los artículos. Se excluyeron de la revisión aquellos trabajos referidos a la carga académica en estudiantes de educación primaria o secundaria, considerando solo aquellos referidos a educación superior. En base a dicha revisión, se realizó una sistematización de los conceptos y dimensiones asociados a carga académica y de los factores que afectan la carga percibida.

Posteriormente, se realizaron dos grupos focales que contaron con la participación de estudiantes de distintas carreras y niveles de la FMUCH. Su objetivo fue conocer, desde las experiencias de los participantes, cuáles son los factores que afectan su percepción de carga académica. Los grupos 
focales contaron con una muestra estructural, considerando como variables principales para la elección de participantes la carrera cursada, el nivel de estudio y la estimación de carga académica que arrojaban mediciones anteriores de la institución para cada nivel de estudio y carrera (Anexo 1$)^{27}$. El análisis de los grupos se realizó por medio de la técnica de análisis de contenido cualitativo con el software Atlas.ti, haciendo uso del modelo de categorías deductivas, que definieron aspectos relevantes de la carga académica percibida ${ }^{28}$.

En base a lo arrojado por la revisión bibliográfica y los grupos focales, se construyeron las matrices de operacionalización del concepto de carga académica, y de los factores que explican la percepción de esta, correspondientes a las Tabla
1 y Tabla 2, respectivamente, a partir de las que se diseñó el instrumento de medición de carga académica.

Este instrumento fue sometido a tres rondas de pretest cualitativos que contaron con la participación de estudiantes de las ocho carreras de la facultad, para mejorar la comprensibilidad general del instrumento ${ }^{36}$. Los estudiantes que respondieron el pretest, además, tuvieron la opción de expresar por escrito sus dudas e impresiones en relación al cuestionario.

Para evaluar la validez de contenido del cuestionario resultante, se realizó una validación por expertos. Catorce especialistas nacionales e internacionales fueron elegidos utilizando como criterio su vinculación a líneas de trabajo en edu-

Tabla 1. Operacionalización del concepto de carga académica

\begin{tabular}{|c|c|c|c|}
\hline Dimensiones & Subdimensiones & \multicolumn{2}{|l|}{ Indicadores } \\
\hline \multirow{4}{*}{$\begin{array}{l}\text { Carga académica } \\
\text { declarada (Prevista) }\end{array}$} & Créditos & \multicolumn{2}{|c|}{ Número de créditos por curso ${ }^{14}$} \\
\hline & Duración del curso & \multicolumn{2}{|c|}{ Número de semanas de duración del curso semestral ${ }^{16,29}$} \\
\hline & $\begin{array}{l}\text { Asignación de horas } \\
\text { presenciales/No presenciales }\end{array}$ & \multicolumn{2}{|c|}{$\begin{array}{l}\text { Número de horas presenciales semestrales contempladas para el } \\
\text { curso }^{16,29}\end{array}$} \\
\hline & & \multicolumn{2}{|c|}{$\begin{array}{l}\text { Número de horas no presenciales semestrales contempladas para } \\
\text { el curso }{ }^{16,29}\end{array}$} \\
\hline \multirow[t]{2}{*}{$\begin{array}{l}\text { Carga académica } \\
\text { real }\end{array}$} & $\begin{array}{l}\text { Carga académica en } \\
\text { modalidad presencial }\end{array}$ & \multicolumn{2}{|c|}{$\begin{array}{l}\text { Número de horas semanales presenciales por curso señaladas por } \\
\text { estudiantes }{ }^{24,30}\end{array}$} \\
\hline & $\begin{array}{l}\text { Carga académica en } \\
\text { modalidad no presencial }\end{array}$ & \multicolumn{2}{|c|}{$\begin{array}{l}\text { Número de horas semanales no presenciales por curso señaladas } \\
\text { por estudiantes } 24,30\end{array}$} \\
\hline \multirow{12}{*}{$\begin{array}{l}\text { Carga académica } \\
\text { percibida }\end{array}$} & \multirow{5}{*}{$\begin{array}{l}\text { Niveles de carga } \\
\text { académica }^{16,19,24}\end{array}$} & \multicolumn{2}{|l|}{ Muy baja } \\
\hline & & \multicolumn{2}{|l|}{ Baja } \\
\hline & & \multicolumn{2}{|l|}{ Adecuada } \\
\hline & & \multicolumn{2}{|l|}{ Alta } \\
\hline & & \multicolumn{2}{|l|}{ Muy alta } \\
\hline & \multirow[t]{7}{*}{ Factores explicativos } & \multirow[t]{2}{*}{ Factores personales ${ }^{26}$} & Factores personales académicos \\
\hline & & & Factores personales no académicos \\
\hline & & \multirow[t]{5}{*}{ Factores institucionales ${ }^{26}$} & $\begin{array}{l}\text { Factores del ambiente de enseñanza- } \\
\text { aprendizaje }\end{array}$ \\
\hline & & & $\begin{array}{l}\text { Relación entre los actores del espacio } \\
\text { educativo }\end{array}$ \\
\hline & & & $\begin{array}{l}\text { Coordinación horizontal de } \\
\text { asignaturas }\end{array}$ \\
\hline & & & Infraestructura \\
\hline & & & Instancias de apoyo institucional \\
\hline
\end{tabular}


Tabla 2. Operacionalización de factores que explican la carga académica percibida

\begin{tabular}{|c|c|c|}
\hline \multicolumn{2}{|c|}{ Tipo de factores } & Indicadores \\
\hline \multicolumn{3}{|c|}{ Factores Explicativos Personales } \\
\hline \multicolumn{2}{|c|}{$\begin{array}{l}\text { Factores personales } \\
\text { académicos }\end{array}$} & $\begin{array}{l}\text { Habilidades/conocimientos previos del estudiante esperados por la asignatura } \\
\text { (Ej: manejo del inglés, ciencias básicas, etc.)* } \\
\text { Hábitos de estudio* } \\
\text { Motivación por la asignatura/carrera* }\end{array}$ \\
\hline \multicolumn{2}{|c|}{$\begin{array}{l}\text { Factores personales } \\
\text { no académicos }\end{array}$} & $\begin{array}{l}\text { Tiempo de traslado* } \\
\text { Soy madre/padre* } \\
\text { Realizo trabajo remunerado* } \\
\text { Vivo sin mis padres o tutor responsable* } \\
\text { Soy de otra región* } \\
\text { Tengo problemas de salud crónicos o relevantes durante el semestre (incluye condi- } \\
\text { ciones médicas y psicológicas)* } \\
\text { Hago actividades extracurriculares (Ej: deportivas, representación estudiantil, artísticas } \\
\text { culturales, etc.)* } \\
\text { No hago actividades extracurriculares (Ej: deportivas, representación estudiantil, } \\
\text { artísticas culturales, etc.)* }\end{array}$ \\
\hline \multicolumn{3}{|c|}{ Factores Explicativos Institucionales } \\
\hline \multirow[t]{2}{*}{$\begin{array}{l}\text { Factores del } \\
\text { ambiente } \\
\text { enseñanza } \\
\text { aprendizaje }\end{array}$} & Docencia & $\begin{array}{l}\text { Habilidades pedagógicas del docente/equipo docente (Ej: Preparación de clases, } \\
\text { material complementario, retroalimentación) } \\
\text { Manejo de los conocimientos de la asignatura por parte del docente/equipo docen- } \\
\text { te }{ }^{31,32} \\
\text { Metodología del curso (Ej: expositivas, participativas, prácticas, videoclases) }{ }^{33} \\
\text { Organización del curso (Ej: cantidad de contenidos contemplados, coordinación del } \\
\text { equipo docente, obligatoriedad de asistencia a clases/prácticos/turnos, etc. })^{33}\end{array}$ \\
\hline & Evaluaciones & $\begin{array}{l}\text { Número de evaluaciones de la asignatura } \\
\text { Se evalúan contenidos que no fueron profundizados en clases ni acordados previa- } \\
\text { mente con el docente }{ }^{31,34} \\
\text { Tipo de evaluación de la asignatura (Ej: control, certamen, presentación oral, diná- } \\
\text { micas grupales, otras) } \text { (11,34 }^{31} \\
\text { No hay un criterio de evaluación estandarizado en la asignatura } \\
\text { Otros aspectos de las evaluaciones (Ej: ponderación, carácter reprobatorio, etc.) }\end{array}$ \\
\hline \multicolumn{2}{|c|}{$\begin{array}{l}\text { Relación entre los actores del } \\
\text { espacio educativo }\end{array}$} & $\begin{array}{l}\text { Relación o comunicación entre docentes y estudiantes }{ }^{35} \\
\text { Relación o comunicación entre estudiantes } \\
\text { Relación o comunicación entre la escuela y los docentes }{ }^{35} \\
\text { Relación o comunicación entre la escuela y los estudiantes }\end{array}$ \\
\hline \multicolumn{2}{|c|}{$\begin{array}{l}\text { Coordinación horizontal de } \\
\text { asignaturas }\end{array}$} & $\begin{array}{l}\text { Coordinación entre asignaturas (Ej: evaluaciones, actividades, horarios, traslado entre } \\
\text { sedes, etc.)* } \\
\text { Extensión de las jornadas de clases/prácticas* } \\
\text { Cantidad de asignaturas cursadas en el semestre* } \\
\text { Rigidez de la malla (Ej: atrasos en la malla dificultan la inscripción de asignaturas, } \\
\text { coordinación entre compañeros o procesos de aprendizaje) } \\
\text { Recalendarización académica (por movilizaciones estudiantiles u otros incidentes)* }\end{array}$ \\
\hline \multicolumn{2}{|c|}{ Infraestructura } & $\begin{array}{l}\text { Condiciones de las aulas/laboratorios/centros de práctica para el desarrollo de las } \\
\text { clases }^{31} \\
\text { Espacio, distribución y equipamiento de la biblioteca* } \\
\text { Faltan espacios comunes recreacionales* } \\
\text { La infraestructura de la universidad no es inclusiva con las necesidades de todos los } \\
\text { estudiantes* }\end{array}$ \\
\hline \multirow{2}{*}{$\begin{array}{l}\text { Instancias } \\
\text { de apoyo } \\
\text { institucional }\end{array}$} & Apoyo académico & $\begin{array}{l}\text { Asistencia a ayudantías* } \\
\text { Efectividad de las mentorías/tutorías* }\end{array}$ \\
\hline & $\begin{array}{l}\text { Apoyo psicológico } \\
\text { de la institución }\end{array}$ & Apoyo psicológico por parte de la institución* \\
\hline
\end{tabular}

*Categoría emergente de los grupos focales. Los indicadores consideraron la revisión bibliográfica y los grupos focales. Sin embargo, la tabla señala la fuente principal para la construcción de cada indicador. 
cación, educación superior, educación en ciencias de la salud, currículum o procesos de validación de instrumentos.

Este análisis se construyó a partir de una pauta de validación que evaluaba cada una de las preguntas de la Encuesta de Carga Académica 2018, en base a los criterios: "Pregunta esencial", "Pregunta adecuada, pero no esencial" o "Pregunta no adecuada". Además, se les solicitó agregar comentarios generales para cada uno de los ítems. Al final de la pauta se agregó una sección para observaciones complementarias de carácter general.

La validez del instrumento se analizó utilizando la razón de validez del contenido (Content Validity Ratio [CVR]) y el índice de validez del contenido (Content Validity Index [CVI]), en la versión de Tristán-López ${ }^{37}$. Dicho análisis de consenso entre panelistas se escogió debido a que no depende del número de jueces participantes.

\section{Resultados}

El instrumento final (Anexo 2) consiste en un cuestionario diseñado para ser respondido de manera autoaplicada a través de formularios electrónicos. Esta decisión se fundamenta en los costos asociados a la aplicación mediante encuestadores y a la flexibilidad horaria que permite, adaptándose a la realidad de las y los estudiantes de carreras de salud.

La encuesta se divide en dos secciones. Tras la pregunta por el consentimiento para participar en la encuesta, la primera sección consta de diez ítems (pregunta 2-11). Estos hacen referencia a la caracterización sociodemográfica general del estudiante y a situaciones personales relacionadas con la problemática de estudio, como el eventual padecimiento de enfermedades crónicas, horas de trabajo remunerado que realiza, tiempo de traslado a la universidad, hijos que tiene bajo su cuidado, personas con las que vive en el hogar y actividades extracurriculares realizadas.

La segunda sección busca medir tanto la carga académica de cada asignatura (preguntas 12-16), como del semestre en su totalidad (preguntas 17 y 18). Por cada una de las asignaturas cursadas en el semestre (obligatorias y electivas), se solicita al participante responder las preguntas 12 a la 16 todas las veces necesarias hasta completar todas las asignaturas del estudiante. Las preguntas $17 \mathrm{y}$ 18 se responden una única vez.

Dentro de las preguntas que se repiten por asignatura, se solicita, en primer lugar, indicar el nombre de la misma y el número de horas semanales dedicadas a esta. Posteriormente, se pide al estudiante caracterizar la carga de la asignatura de acuerdo a su percepción, a través de una escala Likert de cinco niveles (muy baja, baja, adecuada, alta, muy alta) y escoger los tres factores que más afectan esta percepción, sin jerarquizarlos. Finalmente, se consulta por la percepción de carga asociada al semestre en general y los cinco factores más importantes asociados a esta.

$\mathrm{El}$ instrumento permite obtener estimaciones de la carga académica real de un estudiante en horas totales por semana, que pueden ser contrastadas con la carga declarada en sus planes de estudio. Estos resultados pueden ser agregados para obtener la carga real promedio del semestre (por nivel o por carrera), o bien, para obtener la carga real promedio de una asignatura. Asimismo, es posible obtener la carga académica percibida del semestre de un estudiante directamente a partir de la encuesta, o bien, la mediana de la carga de un estudiante, del semestre o de una asignatura. Los factores que influyen en la carga académica real y percibida pueden ser analizados de acuerdo a su frecuencia en los mismos niveles de análisis. Para más detalles, ver Anexo 3.

\section{Validación del instrumento}

La Tabla 3 evidencia los valores de CVR y CVI por pregunta o ítem, por sección y para el instrumento completo. El número de validadores varía en cada ítem, dado que no todos respondieron la pauta de validación completa.

Todos los ítems considerados en la primera sección fueron aceptados, ya que los CVR de cada uno de estos supera el límite establecido de acuerdo al número de evaluadores. El CVI correspondiente al promedio de toda la sección fue de 0,77 , estando sobre el 0,58 establecido como mínimo. Esto indica que existió $77 \%$ de consenso entre los jueces respecto a la suficiencia de los ítems de la primera sección del cuestionario.

Asimismo, todos los ítems considerados en la segunda sección fueron aceptados, ya que los CVR de cada uno de ellos superó el límite establecido de acuerdo al número de evaluadores. El CVI para esta sección del cuestionario indicó que existió 
Tabla 3. Valores de CVR y CVI de la Encuesta de Carga Académica por ítem, sección e instrumento completo

\begin{tabular}{|c|c|c|c|c|c|c|}
\hline \multirow{2}{*}{$\begin{array}{l}\text { Sección de la } \\
\text { encuesta }\end{array}$} & \multirow[t]{2}{*}{ Ítem } & \multirow{2}{*}{$\begin{array}{c}\text { n de } \\
\text { jueces }\end{array}$} & \multirow[t]{2}{*}{ Acuerdos } & \multicolumn{3}{|c|}{ Validez de contenido (Tristán - López) } \\
\hline & & & & CVR & $\begin{array}{l}\text { CVI (aceptados) } \\
\text { por sección }\end{array}$ & CVI (aceptados) \\
\hline \multirow{10}{*}{$\begin{array}{l}\text { Primera Sección: } \\
\text { Caracterización }\end{array}$} & 2 & 14 & 9 & 0,64 & \multirow{10}{*}{0,77} & \\
\hline & 3 & 14 & 12 & 0,86 & & \\
\hline & 4 & 14 & 10 & 0,71 & & \\
\hline & 5 & 14 & 11 & 0,79 & & \\
\hline & 6 & 14 & 10 & 0,71 & & \\
\hline & 7 & 14 & 11 & 0,79 & & \\
\hline & 8 & 14 & 12 & 0,86 & & \\
\hline & 9 & 14 & 10 & 0,71 & & \\
\hline & 10 & 14 & 10 & 0,71 & & 0,84 \\
\hline & 11 & 14 & 13 & 0,93 & & \\
\hline \multirow{7}{*}{$\begin{array}{l}\text { Segunda Sección: } \\
\text { Medición de carga }\end{array}$} & 12 & 14 & 14 & 1,00 & \multirow{7}{*}{0,94} & \\
\hline & 13 & 13 & 13 & 1,00 & & \\
\hline & 14 & 14 & 13 & 0,93 & & \\
\hline & 15 & 13 & 10 & 0,77 & & \\
\hline & 16 & 13 & 12 & 0,92 & & \\
\hline & 17 & 12 & 12 & 1,00 & & \\
\hline & 18 & 14 & 13 & 0,93 & & \\
\hline
\end{tabular}

CVR: Content Validity Ratio. CVI: Content Validity Index.

94\% de consenso entre los jueces respecto a la suficiencia de los ítems que lo componen.

Finalmente, utilizando la misma prueba para el instrumento completo, se aceptó 100\% de los ítems del cuestionario. El índice de validez de contenido para el instrumento indica que existió $84 \%$ de consenso entre los jueces respecto a la suficiencia de los ítems del cuestionario. Pese a que todos los ítems fueron validados, se decidió recoger comentarios referidos a la claridad de los enunciados, por lo que la redacción original de algunos de estos fue modificada.

\section{Discusión}

El presente trabajo tuvo como propósito diseñar y validar un instrumento para medir la carga académica real y percibida en estudiantes de educación superior en el área de la salud. El instrumento resultante fue un cuestionario que consta de dos secciones con 17 ítems en total, validado por contenido mediante el juicio de 14 expertos, utilizando el índice propuesto por Tristán-López ${ }^{37}$.

Dado que el cuestionario se validó en su totalidad, es posible afirmar que este instrumento constituye un aporte para la medición de la carga académica real y percibida de estudiantes de carreras del área de la salud. Además, su utilización ofrece la posibilidad de conocer los factores que influyen en la percepción de carga académica, aportando elementos para entender la relación entre las cargas académicas declarada, real y percibida.

Si bien se han utilizado distintos tipos de instrumento para estudiar la carga académica, el carácter autoaplicado del cuestionario propuesto ofrece la posibilidad de una implementación sencilla y de bajo costo ${ }^{5,23,38-40}$. Gracias a ello, puede ser aplicado a un gran número de estudiantes, entregando un amplio volumen de datos fácilmente sistematizables. Otra ventaja que ofrece el instrumento es que permite una evaluación tanto de la carga académica a nivel de asignatura, como a nivel de semestre; asimismo, permite realizar un 
análisis tanto a escala de facultad como a nivel de grupos de interés, haciendo posible la generación de perfiles de estudiantes. Todos estos aspectos lo perfilan como una herramienta que puede aportar a la gestión, planificación y evaluación de los planes de estudio y programas de acompañamiento a estudiantes del área de la salud.

Pese a ello, es necesario considerar que este instrumento se formuló dentro de la FMUCH, por lo que su aplicabilidad en otros contextos podría requerir eventuales adaptaciones de los factores de carga académica percibida, especialmente en unidades académicas no relacionadas con el área de la salud. Además, en caso de no contar con bases de datos institucionales que contengan información sociodemográfica robusta de los estudiantes, podría ser necesario incorporar preguntas de caracterización que complementen los datos disponibles. Finalmente, cabe considerar que el instrumento fue diseñado para aplicarse en un contexto de educación presencial, por lo que no contempla factores de carga académica específicos de la enseñanza remota.

Respecto al método de validación seleccionado, se considera que aporta observaciones valiosas para adecuar el instrumento, facilitando su comprensión. Esto último es de suma importancia considerando su naturaleza autoaplicada, que impide a los estudiantes resolver dudas al no contar con la presencia de un encuestador.

En conclusión, la importancia de este instrumento radica en que permite obtener datos necesarios para la toma de decisiones en gestión y evaluación en educación en salud, aportando a combatir los problemas asociados a la sobrecarga académica. Resulta relevante que la implementación de la encuesta tenga periodicidad para que permita la comparación entre cohortes de estudiantes. Esto posibilitará observar el progreso de los planes de apoyo estudiantil y el efecto de las políticas de reestructuración curricular.

Agradecimientos: Agradecemos a la Facultad de Medicina de la Universidad de Chile y a las y los estudiantes que participaron voluntariamente en los pretest y en los grupos focales; a las y los expertos, que, con su juicio y comentarios, contribuyeron a la validación de este instrumento; a Patricio Paredes, Diego Vidal, Áurea Argomedo, Margarita Reyes, Valentina Reyes, Viviana Arévalo y Sebastián Buzeta por su colaboración con el equipo de investigación.

\section{Referencias}

1. Bowyer K. A Model of Student workload. J High Educ Policy Manag. 2012; 34 (3): 239-58.

2. Chambers E. Work-load and the Quality of Student Learning. Stud High Educ. 1992; 17 (2): 141-53.

3. Kember D, Jamieson QW, Pomfret M, Wong ETT. Learning Approaches, Study Time and Academic Performance. High Educ. 1995; 29 (3): 329-43.

4. Kember D, Ng S, Tse H, Wong ETT, Pomfret M. An Examination of the Interrelationships between Workload, Study Time, Learning Approaches and Academic Outcomes. Stud High Educ. 1996; 21 (3): 347-58.

5. Kember D, Leung DYP. Influences upon Students' Perceptions of Workload. Educ Psychol. 1998; 18 (3): 293-307.

6. Kyndt E, Dochy F, Struyven K, Cascallar E. The perception of workload and task complexity and its influence on students' approaches to learning: A study in higher education. Eur J Psychol Educ. 2011; 26 (3): 393-415.

7. Azimi H, Shams J, Sohrabi M, Malih N. The frequency of academic burnout and related factors among medical students at Shahid Beheshti University of Medical Sciences, Tehran, in 2016. Soc Determ Heal. 2016; 2 (1): 21-8.

8. Castillo C, Chacón de la Cruz T, Díaz-Véliz G. Ansiedad y fuentes de estrés académico en estudiantes de carreras de la salud. Investigación Educ Médica 2016; 5 (20): 230-7.

9. Preciado-Serrano M, Vázquez-Goñi J. Perfil de estrés y síndrome de burnout en estudiantes mexicanos de odontología de una universidad pública. Rev Chil Neuro-psiquiatr. 2010; 48 (1): 11-9.

10. Solomon P, Finch E. A Qualitative Study Identifying Stressors Associated With Adapting to Problem-Based Learning. Teach Learn Med. 1998; 10 (2): 58-64.

11. Durán F, Azevedo W. Relação entre a carga horária e qualidade de vida dos alunos do curso medicina de uma faculdade de Salvador. Rev Bras de Neurol Psiquiatr. 2019; 23 (3): 206-22.

12. Pogacnik M, Juznic P, Kosorok-Drobnic M, Pogacnik A, Cestnik V, Kogovsek J, et al. An attempt to estimate students' workload. J Vet Med Educ. 2003; 31 (3): 25560.

13. Mujica P, Prieto JP. Sistema de Créditos Transferibles y Carga de Trabajo de los Estudiantes en las Universidades del Consejo de Rectores. Calid Educ. 2007; (26): 293306.

14. Ortiz V, Jenaro C, García J, Zubiauz M, Mayor M, Arana J. Carga de Trabajo en el EEES: La necesidad de coordinación docente entre asignaturas. En: Tortosa Ybañez MT, Álvarez Teruel JD, Rellín Baudel N, Editores, Actas 
de las IX Jornadas de Redes de Investigación en Docencia Universitaria. España: Universidad de Alicante 2011; 1863-77.

15. McKay R. Effectiveness of learning: the place of study. En: Warren Piper D, Editor, The efficiency and effectiveness of teaching in higher education. Londres: University of London, Institute of Education 1978; 86-94.

16. La Palio L. Time study of students and house staff on a university medical service. J Med Educ. 1981; 56 (1): 61-4.

17. García A, García-León J. Una experiencia de medición de la carga de trabajo percibida por los estudiantes para facilitar la coordinación horizontal. Rev Docencia Univ. 2017; 15 (1): 81-104.

18. Kyndt E, Berghmans I, Dochy F, Bulckens L. "Time is not enough.” Workload in higher education: A student perspective. High Educ Res Dev. 2014; 33 (4): 684-98.

19. Hounsell DJ. Contrasting Conceptions of Essay-writing. En: Mraton F, Hounsel D, Entwistle N, Editores, The Experience of Learning: Implications for teaching and studying in higher education. Edinburgo: Scottish Academic Press 1984; 106-25.

20. Ramsden P. Improving Teaching and Learning in Higher Education: The case for a relational perspective. Stud High Educ. 1987; 12 (3): 275-86.

21. López-Núñez M, Rubio-Valdehita S, Díaz-Ramiro E. Cambios producidos por la adaptación al EEES en la carga de trabajo de estudiantes de Psicología. Rev Esp Pedagog. 2019; 77 (272): 163-76.

22. Souto-Iglesias A, Baeza M. A probabilistic approach to studentworkload: empirical distributions and ECTS. High Educ. 2018; 76 (6): 1007-25.

23. Kember D. Interpreting student workload and the factors which shape students' perceptions of their workload. Stud High Educ. 2004; 29 (2): 165-84.

24. Kember D, Leung DYP. Characterising a teaching and learning environment conducive to making demands on students while not making their workload excessive. Stud High Educ. 2006; 31: 185-98.

25. Kyndt E, Dochy F, Struyven K, Cascallar E. The direct and indirect effect of motivation for learning on students' approaches to learning through the perceptions of workload and task complexity. High Educ Res Dev. 2011; 30 (2): 135-50.

26. Scully G, Kerr R. Studentworkload and assessment: Strategies to manage expectations and inform curriculum development. Account Educ. 2014; 23 (5): 443-66.

27. Canales, M. El grupo de discusión y el grupo focal. En: Canales Cerón M, editor, Metodologías de Investigación Social. Santiago, Chile: LOM ediciones 2006; 265-87.

28. Andréu J. Las técnicas de Análisis de Contenido: Una revisión actualizada [Internet]. Documento en línea
1998. Disponible en: http://mastor.cl/blog/wp-content/uploads/2018/02/Andreu.-analisis-de-contenido.-34-pags-pdf.pdf

29. López-Pastor V, Pintor P, Muros B, Webb G. Formative assessment strategies and their effect on student performance and on student and tutor workload: the results of research projects undertaken in preparation for greater convergence of universities in Spain within the European Higher Education Area (EHEA). J Furth High Educ. 2013; 37 (2): 163-80.

30. Galvan T, Branco G, Saurin T. Avaliação de carga de trabalho em alunos de pós-graduação em engenharia de produção: um estudo exploratório. Gest prod. 2015; 22 (3): 678-90.

31. Díaz F, Hernández G. Estrategias docentes para un aprendizaje significativo. México: McGraw-Hill; 2002.

32. Gutiérrez-Provecho ML, López Aguado M. Relación de los enfoques de aprendizaje con el rendimiento y la estimación del tiempo dedicado por los estudiantes en la realización del prácticum. Bordón. Revista de pedagogía 2013; 65 (3): 25-37.

33. Fernández N. Fundamentos del proceso educativo enseñanza, aprendizaje y evaluación a distancia. RIED. 2014; 17 (2): 75-93.

34. Medina M. Influencia de la interacción alumno-docente en el proceso enseñanza-aprendizaje. Paakat: Rev Tecnol Soc. 2015; 5 (8). Disponible en: http://www.udgvirtual. udg.mx/paakat/index.php/paakat/article/view/230/347 [Consultado el 22 de diciembre de 2020].

35. Martínez-Figueira M, Páramo-Iglesias M, De Matos Eugénia. Desafíos actuales a la inclusión: un estudio de caso en un aula de preescolar portuguesa. Rev Actual Investig Educ. 2015; 15 (1): 621-39.

36. Asún R. Construcción de Cuestionarios y Escalas: El Proceso de la Producción de Información Cuantitativa. En: Canales Cerón M, editor, Metodologías de Investigación Social. Santiago, Chile: LOM ediciones 2006; 63-113.

37. Tristan-López A. Modificación al modelo de Lawshe para el dictamen cuantitativo de la validez de contenido de un instrumento objetivo. Av en Medición 2008; 6 (1): $37-48$.

38. Scott SV. Quantifying the assessment loads of students and staff: the challenge of selecting appropriate metrics. J Furth High Educ. 2015; 39 (5): 699-712.

39. Castellón-Zelaya LA. Análisis del proceso de medición de cargas de trabajo en el Instituto Nacional de Aprendizaje de Costa Rica. Inter Sedes 2017; 18 (38): 88-114.

40. Diseth A. Students' evaluation of teaching, approaches to learning, and academic achievement. Scand J Educ Res. 2007; 51 (2): 185-204. 